Fig. 2. Kleinstes von mir gesehenes Individuum (Embry) von Rhabditis genitalis. (Dieselbe Vergrösserung.)

Fig. 3. Geschlechtloses Individuum von Rbabditis genitalis. (0c. 3. 0bj. 2 Verick. Vergrösserung 120.)

Fig. 4. Eier eines Weibchens von Rhabditis genitalis. (Oc. 1. 0bj. 7 Verick. Vergrösserung 250.) Das unter $B$ gezeichnete zeigt das vollendete erste Furchungsstadium.

Fig. 5. Das hinterste Körperende eines Männchens von Rhabditis genitalis (a). b Der Schwanztheil desselben, der durch eine lancetförmige Chitinlamelle umsäumt wird (c); diese zeigt zu beiden Seiten strahlfôrmige Bippen. dd Die beiden Spicula. e Chylusdarm. f Samenkanälchen. (Oc. 2. 0bj. A Verick. Vergrösserung 170.)

Fig. 6 u. 7. Dasselbe wie Fig. 5. a Chylusdarm. b concaver Ausschnitt des Schwanztheiles; in Fig. 7 mit Chitinbaaren besetzt. c Beide Spicula, d Samenkanälchen. (Oc. 1. Obj. 7 Verick. Vergrösserung 250.)

\title{
IX.
}

\section{Woraus besteht der scliwarze Punkt der Comedonen?}

\author{
Von Dr. P. G. Unna in Hamburg.
}

"Uns bleibt ein Erdenrest

Die meisten Leser werden ohne Zweifel sofort mit der Gegenfrage bei der Hand sein: „nun, woraus denn anders als aus Schmutz?"6

Wie berechtigt diese Gegenfrage sei, dessen wird man inne, wenn man auf diesen Punkt hin die Meinungen der Lehrbïcher dieses Jahrhunderts durchgeht, welche mit bestechender Einstimmigkeit eine, wie ich gleich voraussehicken will, dennoch grundfalsche Auffassung vertreten.

Der erste, welcher in der schwarzen Färbung einen Einfluss der Atmosphäre walten sieht, ist Alibert ${ }^{1}$ ):

"l'humeur onctueuse, filtrée par les petits canaux cutanés, se concrète et noircit par l'action de l'air atmosphérique".

1) Altbert, Monographie des dermatoses. 1835. T. II. p. 62. 
In ähnlich untestimmter Weise drückte Bateman sich aus, während Sam. Plumbe, Green, Cazenave und Schedel, Gibert die' Aetiologie der Schwarzfärbung ganz unberührt liessen.

Auf diese Autoren folgte Fuchs ${ }^{1}$ ) mit folgender, positiver Angabe:

„Die ausgezogene Masse ..... deren im Ostium gelegenes Ende durch anklebenden Schmutz u. dergl. dunkel gefärbt wurde."

Ihm folgte Erasm. Wilson (1846). Seit dieser Zeit zieht sich die falsche These durch fast alle Lehrbücher hindurch, besonders seit G. Simon and v. Bärensprung ibre gewichtigen Stimmen auch für dieselbe in die Wagschale warfen. Simon, der bekanntlich den Comedonen seine volle Aufmerksamkeit schenkte, sprach sich zwar zweifelnd über die Natur der Färbung aus, aber doch in einer Weise, dass die an das Vorhandensein von Schmutz Glaubenden durch seinen Ausspruch in ihrer Ansicht bestärkt werden konnten. v. Bärensprung ${ }^{2}$ ) dagegen äussert ganz apodiktisch:

„Die schwarze Färbung ihres sog. Kopfes rührt sicher nur von Schmutztheilen her."

So finden sich denn von nun an kaum noch leise Zweifel ausgesprochen, wie bei Zeiss( ${ }^{3}$ ), welcher die Anomalien der Drüsensecretion in der ersten Auflage (1860) des Hebra'schen Handbuches bearbeitete, indem er als Inhalt der Comedonen u. A. braune Körperchen angiebt und hinzufügt:

"wahrscheinlich die von aussen in den Follikel gelangten Schmutztheile.“

Kleinhans (1866) und selbst Locher (1867), der so bestrebt ist, bei keiner Sache in verba magistri zu schwören, reden vom Schmutze der Comedonen in makro- und mikroskopischer Hinsicht als von einer erledigten, gleichsam selbstverständlichen Angelegenheit.

Endlich wurde diese Lehre von Rindfleiseh $\left.{ }^{4}\right)$ :

"In Wabrheit schaute dieses Ende aus der Haartasche hervor und wurde durch Schmutz so schwarz gefärbt."

1) Fuchs, Die krankhaften Veränderungen der Haut. 1840. Bd. I. S. 223.

2) v. Bärensprung, Beitr. z. Anat. u. Phys. d. menschl. Haut. 1848.

3) Zeissl, in Hebra, Handb. d. acuten Exantheme u. Hautkrankh. 1860. S, 66.

4) Rindfleisch, Lehrb. der pathol. Gewebelehre. 2. Aufl. 1871. S. 283. 
und Virchow ${ }^{x}$ :

„An ihrer Spitze haben sie meist einen schwärzlichen oder . bräunlichen, durch Schmutzfärbung hervorgebrachten Theil“" sanctionirt und daher ist es nicht wunderbar, dass die neuesten Lehrbücher von Kaposi (1879) und Bebrend (1879) die vor 40 Jahren von $F u c h s$ aufgestellte Lehre, fast immer mit denselben Ausdrücken, wiederholen. Bemerkenswerth erscheint es deshalb immerbin, dass J. Neumann ${ }^{2}$ ) keine solche Angabe macht und dass Hebra in der zweiten Auflage seines Werkes, in dem von ihm selbst bearbeiteten Artikel über die Comedonen etwas kurz aber sehr richtig bemerkt:

„sind an ihrew zu Tage sehenden Ende verschieden gefärbt" -

- und nicht von Schmutz redet.

Längst, ehe ich durch die mikroskopische Untersuchung der Comedonen mir ein Urtheil über die sog. Schmutztheilchen bilden konnte, stand es bei mir nach der einfachen klinischen Beobachtung fest, dass diese hergebrachte Ansicht irrthümlich sein müsse. Besonders seit durch die Empfehlungen von Volkmann, Auspitz und Ellinger die mechanische Behandlung der Acne ganz in den Vordergrund getreten, ist der behandelnde Arzt weit mehr in der Lage wie früher, die Comedonen unter seinen Augen entstehen zu sehen.

Man nehme z. B. eine Acne punctata von der wenig entzündlichen, möglichst torpiden Form. Schabt man hier die befallene Hautpartie mit langsamen aber kräftigen Zügen ab, so sieht man in einigen Fällen schon nach den ersten Zügen des Löffels, sicher jedoch nach wiederholten Abreibungen, dass einige Comedonen zugleich mit den abgeschabten Hornblậttchen vollständig herausgehoben werden. Es sind dies die ältesten, deren Köpfe die Hornschicht bereits über das Hautniveau emporwölbten.

Mit der scharfen Kante des Löffels hat man diese Kuppe von Hornzellen, welche zuweilen auch bereits schwärzlich gefärbt ist, resecirt und den daran hängenden Comedo mitgerissen. Die meisten Mitesser folgen jedoch dem scharfen Löffel nicht ohne Weiteres,

1) Virchow, Geschwülste. 1863. Thl. I. S. 216.

2) Mir liegen die Auflagen seines Lehrbuches von 1870 (2te) und 1880 (5te) vor. 
aber die Abschabung hat doch den Effect, die normal weisse Hornkuppe, die sie bedeckt, wie einen Deckel fortzuheben, so dass der Comedo, resp. sein schwarzer Kopf, jetzt erst etwas unter dem allgemeinen Niveau sicbtbar wird.

Auf diese Weise entdeckt man viele Comedonen erst während des Abschabens unter der ungefärbten Hornschicht. Für alle diese - und ihre Zahl ist nicht klein wäre also die Vorstellung, dass der Schmutz von aussen herrühre, nur unter einer zweiten willkürlichen Annahme haltbar, dass nehmlich, nachdem von aussen Schmutz in die offene Talgdrüse gelangt, die allgemeine Hornschicht wieder über diesem Fremdkörper zusammengewachsen wäre. Man bemerkt aber weiter ebenso häufig Stellen, an denen Hornlamellen abgeschabt sind, im Centrum der so entstandenen Grübchen bereits leicht gelbliche Pfröpfe, die am nächsten Tage sich bräunlich färben, indem sie weiter zur Oberfläche rücken und nach ein paar Tagen zu gewöbnlichen, schwarzen, etwas vorspringenden Mitessern geworden sind. Auch hier liegt der Einwurf nur allzunahe, es möchte der zuerst gelbliche Punkt durch Schmutz nachträglich schwarz gefärbt sein. Aber wie viel künstlicher ist diese Erklärung als die andere, dass die bereits unter der Hornschicht entstandene Gelbfärbung nur das erste Stadium der Schwarzfärbung ist, in welche sie sich mit oder ohne Berührung der Atmosphäre in kürzerer oder längerer Zeit nothwendig umwandelt.

Man kann auch leicht beobachten, dass gewisse Medicationen von grossem Einfluss auf die Entstehung der schwarzen Punkte sind. Vor allem muss ich hier die vielgebräuchlichen Schwefelmittel erwähnen, welche die Verfärbung sehr begünstigen. Ich betone aber gleich dabei, dass es sich hier nicht einfach um die oberflächliche Bildung eines schwarzen Schwefelmetalls handeln kann, da man durch andere ,austrocknende“6 Mittel, z. B. weissen Bolus, dasselbe erreicht und zwar kann man auf diesse Weise die schwarzen Punkte gleichsam züchten während einer Behandlung, welche das Gesicht Nachts mit der Maske bedeekt erhält und bei Tage durch Puder oder Salben eine für das Durchdringen von Schmutz möglichst ungünstige Schutzdecke herstellt.

Wenn die Schwarzfärbung wirklich von Schmutz herrübrte, so würden überdies die schwarzen Comedonen das Vorrecht gewisser 
Berufsklassen, der Koblenarbeiter und Dreckfeger sein. Dies ist jedoch nicht der Fall. Ich erinnere hingegen an die allen Collegen bekannte Häufigkeit dieses Uebels grade unter den wohlsituirtesten, etwas chlorotiscben jungen Mädchen.

Desgleichen habe ich nie einen Unterschied zwischen Landund Stadtbewohnern in Bezug auf die Häufigkeit der Acne punctata wahrnehmen können. Auch müsste unter der gewöhnlichen Voraussetzung die Acne des Gesichts weit eher zur Schwarzfärbung der Comedonen Anlass geben als die der Brust und des Rückens. Auch diese Folgerung konnte ich nicht bestätigt finden.

Endlich lebrt die klinische Beobachtung noch, dass die Schwarzfärbung an alten Comedonen nicht nur an der Oberfläche baftet, sondern immer weiter in die Tiefe kriecht. Hin und wieder findet man sehr alte Exemplare, die von hornartiger Consistenz auf 1 bis $2 \mathrm{Mm}$. Tiefe mit abnehmender Intensität geschwärzt sind. Ja, aus der Umgebung von Narben habe ich aus den oft mit zwei Oeffnungen frei mündenden Kanälen der Hornschicht Comedonen von $1 \mathrm{Cm}$. Länge und darüber mit einer feinen Sonde herausgeschoben, die oft Jahre an Ort und Stelle gesessen und vollkommen geschwärzt waren. Ist es aber glaublich, dass Schmutztheile in diese fest in die Hornschicht eingekeilten, schwer unter dem Deckglase zerdrückbaren Massen bis zu solcher Tiefe eindringen können? Wahrlich, wenn schon das Einwandern von Kohlenpartikelchen in das zarte und saftige Gewebe der Lunge genug des Räthselbaften bietet, so stehen wir hier vor etwas noch Unbegreiflicherem. Es stimmt denn schliesslich auch mit diesen Erfahrungen, dass die grösste Reinlichkeit, das peinliche Fernhalten von Schmutz die Schwarzfärbung der Comedonen in keiner. Weise hindert und dass nur ein sehr häufiges Waschen mit scharfen Seifen, welche die Hornschicht in beständiger stärkerer Abschilferung erhält, dieselbe zu beschränken vermag.

Alle diese klinischen und für unsere Frage aprioristischen Gründe wären jedoch völlig hinfällig, wenn denn nun wirklich Schmutztheilchen im schwarzen Kopfe des Mitessers nachgewiesen wären. Aber quod non. Soviel auch stets davon geredet wurde, noch Niemand hat bisher wirklich Schmutz-oder Staubtheilchen an diesem Orte nachweisen können. Alle Untersucher geben übereinstimmend nur das Vorhandensein von ver- 
hornten und verfetteten Epithelien, freiem Fett, Cholestearinkrystallen und Haaren an. Das Vorkommen von sog. Molluscumzellen ist bebauptet, jedoch wieder in Abrede gestellt; auch ich habe die charakteristischen Zellen des sog. subeutanen Condyloms (Molluscum contagiosum, verrucosum) nie in Comedonen angetroffen. Ueber denjenigen Bestandtheil jedoch, welcher die schwarze Färbung bedingt, herrscht völlige Unklarheit. Dass ausser einer diffusen Färbung der Comedonenspitze pigmentirte Körperchen vorkommen, konnte unmöglich einem Untersucher auch bei der primitivsten Methode entgehen. Es fehlt jedoch bisher jede eingehende Untersuchung, um festzustellen, ob es sich bei den dunkelgefärbten Körpern um Pigmentkörner, um Pigmentzellen (Henle), um diffus pigmentirte Hornzellen oder — um die bekannten Bestandtheile des allgegenwärtigen Schmutzes handelt. Ich beginne mit dem letzteren.

Ein Jeder weiss, was Schmutz mikroskopisch zu bedeuten hat, es ist ja einfach ein Sediment aller in der Atmosphäre aufgeschwemmten, festen Körperchen. Wir müssen also vor Allem Kohlepartikelchen, dann Sand und Metallkörnchen, feinste Fragmente von Holzfasern, Seiden-, Leinen- und Baumwollenfäden, Haaren, Federn und Epidermisschuppen, kleinste Theilchen von bearbeiteten Zeugen, Leder und Papier vorfinden. Zu diesen universellen Bestandtheilen des Schmutzes kommen dann an verschiedenen Localitäten die eigenthümlichsten Zusätze, besonders in Fabriken, Lagerräumen, Apotheken, Droguerien u. s. w., während auf dem Lande Theile von frischen Pfianzen und Sandkörnchen gegenüber der Kohle vorwiegen. Alle letztgenannten localen Vorkommnisse können wir bei den Comedonen als einem universellen Uebel vernachlässigen. Nicht so die ersterwähnten und ich stelle an denjenigen, der die Anwesenheit von Schmutz in den Comedonen behauptet, die wohlberechtigte Forderung, dass er einen regelmässigen oder wenigstens sehr häufigen Befund etwa von Baumwollenfasern, Federn oder Kohlepartikelehen, genug von solchen Bestandtheilen des Schmutzes erweise, welche überall vorhanden und mit Sicherheit als Producte des menschlichen Körpers auszuschliessen sind. Dann erst würde die alte Theorie von der Schmutzfärbung der Comedonen und die ebenso wenig berechtigte von der Verstopfung der Talgdrüsen durch Schmutz eine sichere 
Grundlage erhalten, während einem seltenen derartigen Vorkommen - das will jch nicht unterlassen auszusprechen - keine wesentliche Bedeutung für die Comedonen beigemessen werden könnte, etwa ebenso wenig, wie wir eine solche beutzutage dem Acarus folliculorum zuschreiben.

Ich habe nun bei vielen bunderten mikroskopisch und mikrochemisch untersuchter Mitesser nicht in einem Falle jene oben genannten universellen Schmutzbestandtheile und besonders die in dieser Richtung so werthvollen, dem Menschen ganz fremden Theile von Pflanzen, Vögeln und bearbeiteten Zeugen auffinden können, obgleich ich stets darauf gefasst war, gelegentlich auch einen solchen Fund zu machen. Natürlich hat man bei diesen Untersuchungen stets Sorge zu treffen, dass die von der Haut des Patienten bis zum Objectträger sich stets mit wirklichen Schmutzbestandtheilen bedeckenden Comedonen äusserlich vorher gereinigt werden. Ich habe das durch Schütteln derselben mit destillirtem Wasser, Alkohol oder Aether im Reagensglase stets auf leichte Weise erreicht, bei welcher Reinigung selbstverständlich der schwarze Kopf der Comedonen sein Ausseben in keiner Weise veränderte. Allerdings nehme ich bei diesem durchaus negativen Resultate die Kohlepartikelchen für's Erste aus, doch nur diese allein und nicht etwa, weil ich solche mit Sicherheit bätte constatiren können, sondern weil dieselben für sich eingehender erörtert $\mathrm{zu}$ werden verdienen. Kohlepartikelchen sind eben nicht durch die gewöhnlichen mikroskopischen Reagentien mit absoluter Sicherheit zu erkennen oder auszuschliessen und ich wende mich daher lieber zu meinem positiven anatomischen und chemischen Befunde, um bei diesem noch einmal auf die Kohlepartikelchen als letzten Zufluchtsort der historischen Idee des Schmutzes zurückzukommen.

Der Comedo besteht, wie das neuerdings sehr richtig und anschaulich von $\mathrm{Kaposi}^{1}$ ) beschrieben ist, aus einem festen Mantel von Hornzellen und einem talghaltigen, weichen Kern. Das äussere Horngerüst kommt besonders schön zur Anschauung, wenn man recht feste, ältere Comedonen in Aether entfettet und dann feine Längsschnitte durch den mittleren Theil anfertigt. Man tropft zu diesem $\mathrm{Zwecke}$ einfach einige Stearintropfen von einem brennenden

1) Pathol. u. Ther. S. 157. 
Licht auf einen Objectträger, drückt dev Comedo in die erstarrende Fettmasse, welche nach völligem Erkalten vom Glase abgehoben und handlich zugeschnitten wird. Man kann dann durch einfaches Abschaben dieses Stearinwürfels die feinsten Comedonenschnitte gewinnen.

Der Bau der Comedonen variirt theils nach ihrem Alter, d. i. der Zeit, die sie an Ort und Stelle gesessen baben, theils nach dem Fettreichthum, in welcher Beziehung der Standort (Nase) von besonderer Wichtigkeit ist, theils endlich je nachdem Entzündungserscheinungen der Follikelwand zugegen waren oder nicht. Nicht jede in wurmförmiger Gestalt aus den Talgdrüsen ausdrückbare Sebummasse ist bereits ein Comedo. Als einen solchen betrachte ich die Talganhäufung nur dann, wenn sie von einer hornigen Schaale entweder ganz oder wenigstens von oben und an den Seiten bis zu einer gewissen Tiefe umgeben ist. Es könnte lächerlich scheinen, einen Comedo so bestimmt definiren zu wollen, urd doch halte ich es für nöthig, da ich in dessen kurzer und nicht uninteressanter Geschichte den wichtigsten Beitrag zur Pathologie der Acne gegeben sehe, über deren Wesen so viele Meinungen aufgestellt sind als Lehrbücher existiren, ohne dass bisher der ganze Prozess eine allseitig befriedigende Aufklärung erhalten hätte.

Ein Comedo beginnt nur dort, wo die Hornschicht über der Mündung einer Talgdrüse glatt hinweggeht, wo mithin ein abnorm fester Zusammenhalt der gesammten verhornenden Epidermis als vorausgehend angenommen werden muss. Reisst man einen Comedo mit der ihn bedeckenden Hornkuppe los, so besteht sein oberster Kopf aus diesen horizontalen Hornschichten, an die sich dann sofort nach unten ein verticaler Horncylinder anlöthel, der von der Stachelschicht des Talgdrüsenausführungsganges herrührt. Dieser ist im Anfange sehr dünn und reicht nicht weit in die Tiefe, so dass der herausgequetschte Comedo zu dieser Zeit mit seinem unteren Ende nackt aus der Hornhülle hervorsiebt. Durch fortwährende Anlagerung neuer Hornschichten vom Ausführungsgange der Talgdrüse auf den seitlichen Hornmantel wird dieser immer dicker und fester und wächst immer weiter in die Tiefe.

Es würde ein solider, den Ausführungsgang erfüllender Horncylinder entstehen, wenn nicht zugleich die Talgproduction der Drüse fortginge. Durch diese wird der früher cylindrische, nach oben 
abgeschlossene Raum allmählich in einen kugligen verwandelt, und zwar müssen wir annehmen, dass diese Production nicht gleichmässig fortgeht, sondern fluthet und ebbt, denn so erklärt es sich am leichtesten, dass der mit Sebum erfüllte Binnenraum nur selten einkammerig, meist mehrfächerig ist, indem Hornzellencomplexe sich zwischen die verschiedenalterigen Sebummassen von der Seite her septenartig eingeschoben haben. Ein solches schliessliches Versiegen der Talgproduction mag denn auch zu endlichem, hornigen Abschlusse des Comedo führen, den wir sehr gewöhnlich, aber nicht immer eintreten sehen. Durch diesen erhält der Comedo nun auch eine untere, hornige Bekleiduug und bildet somit einen eiförmigen, mil dem kugeligen Ende nach unten schauenden Körper mit vollständig glatter, horniger Schale und im Innern Fett und Fettzellen enthaltend, die durch hornige, schrägliegende Septen getrennt werden. Der secretorische Auftrieb des ganzen Gebildes bedingt es, dass das obere Ende, welches die Hornschicht zu sprengen sucht, während es in dieselbe eindringt, zugespitzt und halsartig eingeschnürt wird, wodurch denn schliesslich der Comedo jene typische kurzhalsige, dickbauchige Flaschenform erhält, die die ältesten und festesten Mitesser so häufig aufweisen.

Aus dieser Beschreibung geht hervor, dass die wahre Ursache jeder Comedonenbildung, mag sie für sich oder in Begleitung einer oberflächlichen Dermatitis als Acne auftreten, allein in einer abnorm festen Verhornung der gesammten Oberfläche zu suchen ist. Indem bierdurch zuerst die Talgdrüsenmündung verwächst, kommt es zu einer Anbäufung von Talg und Talgzellen, und durch den Fortschritt dieser Keratose auf den Ausführungsgang der Talgdrüse werden die sich hier abschuppenden Zellen nicht einfach dem Sebum beigemengt, wodurch es zu einem Atherom kommen würde, sondern die Talgmassen werden in grösseren oder kleineren Partien eingekapselt und nach Loslösung der betreffenden Hornschichten von der Wand des Ausführungsganges wird dann diese talghaltige Hornkapsel als ein kleiner isolirter Körper, als ein Comedo, frei.

Ich kann mich also nicht der Ansicht von Bisiadeckj und Kaposi anschliessen, wonach ein seitlich aus der Haartasche in den Ausführungsgang der Talgdrüse hineinragendes Haar durch Anstossen an der gegenüberliegenden Wand hier einen Proliferationsprozess bedingen soll, der den Gang verschliesst, einfach schon 
aus dem Grunde nicht, weil diese schrägliegenden, an der Spitze umgekrümmten Härchen oft zu vielen, bis 30 Exemplaren, in verschiedenen Höhen innerhalb der Comedonen vorkommen, häufig aber gänzlich fehlen, während viele gerade Haare gestreckt im Comedo eingeschlossen sind, weil ferner die Vertheilung der Hornsubstanz im Comedo nicht auf einen Ausgangspunkt, sondern auf eine allgemeine Betheiligung des ganzen Ausführungsganges hinweist, weil weiter dieses seitliche Einschliessen von Haaren in den Comedo hauptsächlich an den grossen, nur selten ganz und auf längere Zeit verschlossenen Drüsenbälgen der Nase vorkommt, die Lieblingsplätze eigentlicher Comedonenbildung jedoch auf Stirn, Wange, Sternum und Rücken, also grade nicht auf der Nase zu suchen sind, weil endlich durch eine solche punctuelle Verschliessung nur ein Atherom, nie aber (ohne Keratose des Ausführungsganges) ein Comedo entstehen könnte. Ueberhaupt gehören die Haare nicht zu den essentiellen Bestandtheilen der Mitesser, sie fehlen sehr oft ganz und sind nach meiner Meinung stets nur passiv dem Drüsensecret beigemengt. Immerhin gewähren sie einiges Interesse, weil ihre Menge, wo eine solche vorhanden ist, einen Schluss auf das Alter des Comedo erlaubt. Freilich darf man nicht alle in einem Comedo befindlichen Lanugohärchen auf einen Haarbalg beziehen, da häufig mehrere an verschiedenen Stellen in einen Ausführungsgang einmünden; aber die dicht und parallel gelagerten Häärchen, die von einem Punkte ausgehen, sind auch sicher aus einem Haarbalg aufgestiegen, und wüsste man bereits die Zeit des hier stattfindenden Haarwechsels, so könnte man das Alter des Comedo ziemlich genau berechnen. Natürlich sehe ich die umgebogene, spiralig aufgerollte und mehrfach geknickte Form vieler Härchen nicht als Ursache, sondern als Folge des Follikelverschlusses an.

Wir kommen, nachdem wir diese anatomische Grundlage gewonnen, zu dem eigentlichen Thema, dem Pigmentgehalte der Comedonen. Man hat hier sicher zwischen zwei Dingen zu unterscheiden, nehmlich der diffusen Pigmentirung der Hornzellen und freien Pigmentkörnern. Gewisse chemische Reactionen sprechen freilich dafür, dass beiden z. Th. dieselben färbenden Substanzen zu Grunde liegen.

Die diffuse Pigmentirung ist in ihrer Vertheilung und Nüancirung sehr geringen Schwankungen unterworfen. Sie nimmt regel- 
mässig die obersten Hornlagen, den sogenannten Kopf des Comedos ein und setzt sich mit abnelumender Intensität am äusseren Mantel desselben fort, um meist schon dicht unter dem Kopfe zu enden. Die Skala der Farben, gelb, braun bis braunschwarz erinnert sehr an die entsprechenden Färbungen der blonden, braunen und schwarzen Haare, soweit dieselben von dem diffusen, nicht körnigen Pigmente derselben herrühren. Es bandelt sich hier also wahrscheinlich nur um verschieden tiefe Nüancirung einer Grundfarbe, die den alternden Hornzellen eigen ist. Da die Spitze des Horngerüstes stets die dunkelste Pigmentirung aufweist, so liegt allerdings der Gedanke nahe, dass die Nähe der Atmosphäre einen begünstigenden Einfluss auf dieselbe ausübe. Man darf aber nicht vergessen, dass die obersten Hornzellén des Mitessers auch zugleich den ältesten Theil desselben darstellen und also möglicherweise in dem tieferen Dunkel der Farbe nur eine Altersveränderung vorliegt.

Im Gegensatze zu diesem diffusen Pigmente bietet das körnige eine grosse Verschiedenheit dar in der Anzahl, Farbe und Grösse der Körner. Nur die Vertheilung ist auch eine ziemlich regelmässige, und zwar halten sich die Körner am meisten im obersten Theile des Comedokernes auf, also dicht unterhalb des nur aus Hornzellen gebildeten Kopfes in einer bereits fetthaltigen Zone. Seltener findet man sie in tieferen Theilen des Kernes, noch spärlicher zwischen den festen Hornlagen des Kopfes und Mantels, hier meist nur nach Bebandlung des Comedos mit Kalilauge.

Dieser einzige Umstand ist bereits geeignet, denjenigen zu enttäuschen, welcher vom Mikroskop einen Aufschluss über die Betheiligung des Schmutzes an der mit blossem Auge sichtbaren Schwärze erwartet. Denn das Mikroskop kann eine solche nur nachweisen, indem es uns gefärbte Körperchen grade an den Stellen zeigt, welche makroskopisch schwarz aussehen - über die Beziehung solcher Körperchen zum äusseren Schmutze müsste dann discutirt werden. So weist das Mikroskop aber an denjenigen Stellen, welche makroskopisch ganz allein die jeweilige Färbung des Comedo bestimmen können, nur diffus pigmentirte Hornzellen nach, während die Pigmentkörner, deren Beziehung zum Schmutz eventuell in Frage kommen kann, in den Comedo eingeschlossen sind und nicht den allergeringsten Einfluss auf die mit blossem Auge sichtbare Färbung besitzen. 
In der Anzahl und Grösse der Pigmentkörner ist keine Regelmässigkeit zu entdecken. Sie kommen in einigen Fällen nur in wenigen Exemplaren, in anderen massenhaft vor; manchmal finden sich nur einige ganz grosse, makroskopisch eben sichtbare Körner, in anderen Fällen wieder viel kleinere, die wie , ein Staub die Zellen bedecken und weiter alle Zwischenstufen $\mathrm{zwischen}$ diesen Extremen.

Der Farbe nach trennen sich die Körner in drei Gruppen, schwarze, braune und blaue. Die braunen und blauen scheinen nur in mittelgrossen und grossen Exemplaren vorzukommen. $O b$ die Farbe der allerfeinsten Körnchen wirklich immer schwarz und nicht bäufig auch braun oder blau ist, wird nicht leicht zu entscheiden sein, da bekanntlich alle feinsten Körperchen im durchgelassenen Lichte schwarz erscheinen, sobald jhre Dimensionen klein sind gegen die Wellenlänge des Lichtes $(0,4-0,6 \mu)$.

Unter diesen Pigmentformen sind wohl hauptsächlich die gröberen, schwarzen Körner von G. Simon und v. Bärensprung für Kohlenstäubchen gebalten worden. Die braunen und blauen Körperchen, die gewöbnlich durchscheinend und dabei von schön gesättigter Farbe sind, scheinen jedoch der Aufmerksamkeit auch dieser Forscher entgangen zu sein, denn es ist nicht anzunehmen, dass sie dieselben gesehen und einfach für Schmutz erklärt haben sollten. Die braunen körperchen habe ich in keinem Comedo bei genauer Untersuchung vermisst, die blauen nur sellten, und zwar nur nach gewissen Medicationen, welche dieselben zu vernichten geeignet waren, wovon später.

Zerquetscht man einen nicht zu alten und festen Comedo unter dem Deckglase und mustert die genannte Zone desselben bei einer 1-200maligen Vergrösserung, so lösen sich die dunklen Stellen in Haufen der beschriebenen Körperchen auf, und indem man einzelne derselben in's Auge fasst, kann man alle chemischen Reactionen unter dem Deckglase mit denselben vornehmen. Da zeigt sich denn zunächst, dass alle Körnerarten sowohl, wie die diffuse Färbung in destillirtem Wasser, Kochsalzlösung, Alkohol, Aether, Chloroform vollkommen unlöslich sind, auch wenn man die Reagentien mil dem Präparat zum Kochen erhitzt. Lässt man dem in Wasser schwimmenden Object unter dem Deckglase Kali-, Natronlösung oder Lig. ammon. caust. zufliessen, so blähen und 
hellen sich alle verhornten Epitelien auf, während alles Pigment ungelöst und unverändert bleibt und daher noch viel deutlicher erscheint als vorher, und eine Menge kleiner Pigmentkörner nun überhaupt erst sichtbar werden. Ich kann die Alkalien deshalb zum Studium der Pigmentformen nur empfehlen. Anders verhält sich das Pigment den starken Mineralsäuren gegenüber. In concentrirter Salz- und Salpetersäure, noch rascher in Königswasser blasst die diffuse Färbung rasch ah, und erhitzt man zum Kochen so lösen sich alle oder nahezu alle Pigmentkörner vollkommen auf. In dieser Reaction liegt der schwerwiegendste, positive Beweis, dass die Schwärze der Comedonen nicht durch Koble hervorgebracht wird. Es ist ein höchst einfacher Versuch, den Jeder an jedem Comedo im Reagensglase bestätigen kann; erhitat man einen solchen in Königswasser zum Kochen, so verschwindet die Färbung augenblicklich. Hat man einen auf diese Weise entfärbten Comedo unter dem Deckglase zerdrückt, so findet man bei vollständigem Mangel der äusseren diffusen Färbung manchmal aber nicht immer im Innern noch einzelne und zwar ganz schwarze Körnchen, die der Säure widerstanden haben, angenommen, dass dieselbe überall in das Innere des Comedo genügend hat eindringen können, was mindestens fraglich bleibt. Immerhin wären diese das Einzige, was Jemand als Kohlepartikelchen ansprechen könnte, wenn auch ihr Ansehen von dem des Russes erheblich verschieden ist. Aber es wird Niemand geneigt sein, auf die Existenz von ein paar inconstanten, erst bei stärkerer Vergrösserung sichtbaren und nicht einmal mit voller Sicherheit mit der Kohle zu identificirenden Stäubchen an der Theorie der Verstopfung der Drüsen durch Schmutz festzubalten, und ich möchte umgekehrt behaupten, dass die vollständige Abwesenheit von Koblenstaub ein an und für sich bemerkenswerthes Phänomen darstellen und darauf hindeuten würde, dass die Talgdrüse schon ehe der Comedo sich bildete, nicht frei mit der Atmosphäre communicirte.

Unter den Pigmentarten widerstehen die blauen Körperchen der Säure am wenigsten. Während das diffuse Pigment und die braunen und schwarzen Körner erst den stärksten Mineralsäuren weichen, verschwinden die blauen Körner bereits in ganz verdünnter Salzsäure, Eisessig, gewöhnlicher (25 procentiger) Essigsäure, schwefliger Säure, ja auch in Lösungen gewisser Salze, wie Alaun, und 
zwar rascher beim Kochen als in der Kälte. Diese Einwirkung der Säuren erstreckt sich auch durch die unversehrte Hornschicht hindurch. Ich habe einen in dieser Beziehung sehr beweisenden Fall beobachtet. Ein junger Mann, Namens R., der mit einer ausgebreiteten Acne punctata des Rückens, der Brust und des Gesichts behaftet, mir im Anfange der Behandlung einen grossen Theil meines Comedonenmaterials mit sehr schön ausgebildeten blauen Körperchen geliefert hatte, zeigte die letzteren nach einer mehrwöchentlichen, erfolgreichen Behandlung mittelst einer Salbe aus Bolus, Glycerin und Essig in den restirenden Comedonen fast gar nicht mehr, während die schwarzen und braunen, freilich auch vermindert, aber doch noch überall zu finden waren. Uebrigens habe ich die blauen Körner sonst überall gefunden, freilich in sehr wechselnder Menge, bei ganz frischen und ganz alten Acneformen, in einzelnen Comedonen auf sonst gesunder Haut, bei Patienten jeden Standes und Alters; ja in zwei kleinen Mitessern vom Hinterkopfe eines gesunden, nur mit Wasser und Seife in Berbihrung gekommenen, viermonatlichen Kindes fand ich neben schwarzem und schön hellbraunem, körnigen Pigment bereits das blaue.

Es würde die Erkenntniss dieser gefärbten Substanzen sehr fördern, wenn dieselben, wie es oft den Anschein hat, Krystallformen erkennen liessen. Dieses ist aber in Wabrheit nicht der Fall. Die geradlinigen Kanten und Winkel, die hin und wieder an ihnen sichtbar werden, sind ganz inconstant und offenbar nur zufällig; am meisten sind rundliche, ganz uncharakteristische Formen vertreten. Bei einer solchen Lage der Dinge wird es für den physiologischen Chemiker wohl nicht so schwer sein, die ungefähre Natur der in Betracht kommenden Verbindungen nach den angegebenen Reactionen zu erschliessen, eine genaue Feststellung derselben dürfte aber, wenigstens bei den schwarzen und braunen Körnern erheblichen Schwierigkeiten unterliegen. Leichter schon werden die blauen Körner zu definiren sein, denn die Auswahl der möglichen chemischen Verbindungen ist hier eine relativ sehr kleine. Ich habe deshalb zunächst den Versuch gewagt, diese blauen Körner mikrochemisch zu bestimmen. Soviel ich sehe, sind dabei die folgenden 8 Gruppen allein in Betracht zu ziehen:

1) Schwefelsaures Kupferoxyd, Kupferlasur, Mineralblau.

2) Phosphorsaures Eisenoxyduloxyd (Vivianit). 
3) Cyaneisenverbindungen (Berlinerblau, Pariserblau).

4) Kobaltverbindungen (Smalte, Kobaltblau).

5) Indigo (Indigcarmin).

6) Anilinblau.

7) Hämatoxylin.

8) Ultramarin.

I. Der Kupfervitriol ist leicht auszuschliessen, da die blauen Körner nicht wie dieser in kaltem und kochendem Wasser löslich sind. Die blauen Kupferverbindungen sind übrigens sämmtlich dadurch auszuschliessen, dass sie nicht, wie die blauen Körner, durch Kochen mit Kupfersulphat eine schwarze Farbe annehmen (s. unten). -

II. Weit plausibler erseheint schon die Annahme, dass das blaue Phosphoreisensalz sich hier ausgeschieden habe, da Phosphorsäure und Eisen in der Asche des Horngewebes, besonders der Haare, reichlich vorkommen, überdies das betr. Salz von Scherer im blauen Schweisse nachgewiesen wurde. Der Vivianit erscheint meist in kleinen, klinorhombischen Krystallen, die häufig zu kugligen, strahligen Bündeln verwachsen; doch findet er sich auch in amorphem Zustande wie die blauen Körner. Durch Salzsäure wird derselbe auch aufgelöst wie dieser, aber die Flüssigkeit färbt sich dabei bräunlich durch Bildung von Eisenchlorid. Schwache Säuren wie Essigsäure, auch Eisessig, verändern dagegen die Vivianitkrystalle nicht und während Kalilauge die blauen Körner unverändert lässt, färbt sie jene rostgelb; dabei zerfallen die strahligen Bündel zuerst, die klinorhombischen Tafeln zuletzt in bräunliche, krümelige Haufen, deren Farbe allmählich schwarzbraun wird. Auch zeigt der Vivianit unter dem Mikroskop durchaus nicht die gesättigt blaue Farbe der Körner, sondern ein mattes Grünblau.

III. Ebenso wie das phosphorsaure Eisensalz können auch die Cyaneisenverbindungen durch ihr Verhalten zu Alkalien mit Sicherheit ausgeschlossen werden, indem bei allen unter Abspaltung von Eisenoxydhydrat Zersetzung und Vernichtung der Farbe eintritt.

IV. Umgekehrt werden Kobaltoxydul und seine blauen Verbindungen durch Säuren nicht entfärbt, während die blauen Körner, wie wir gesehen, durch dieselben rasch zersetzt werden unter Verschwinden der blauen Farbe.

V. Die blauen Körner können auch nicht aus Indigo bestehen, 
da derselbe unter keinen Umständen in Essigsäure löslich ist. Der Indigcarmin (indigblauschwefelsaures Kali) kommt als wasserlösliches Salz ebenfalls nicht in Frage.

VI. Anilinblau löst sich wie die blauen Körner in verdünnten Säuren, jedoch ohne Zerstörung der Farbe. Auch ist es in Alkohol beim Kochen löslich, während jene unlöslich sind.

VII. Hämatoxylin erwähne ich nur der Vollständigkeit halber. Es kann natürlich nicht ernstlich in Betracht gezogen werden, da es, an und für sich braun, erst in alkalischer Lösung blau und durch Salzsäure wieder rotb gefärbt wird, die dabei selbst eine rothe Farbe annimmt.

VIII. Die blauen Körner auf Ultramarin zu untersuchen, wurde ich bereits durch einen mir bekannten Chemiker hingewiesen, welchem ich meine Präparate zu demonstriren Gelegenheit hatte. Nach Ausschliessung aller übrigen blauen Pigmente kam ich denn auch hierauf wieder zurïck und fand in der That, dass sich die Reactionen des Ultramarins, soweit sie sich unter das Mikroskop verlegen lassen, fast vollständig mit denen der blauen Körner decken. Ultramarin ist (nach Ritter) ein Natron-Thonerdesilikat verbunden mit Mehrfach-Schwefelnatrium und wahrscheinlich unterschwefligsaurem, vielleicht auch schwefligsaurem Natron. Es bleibt wie die blauen Körner unverändert in kaltem und kochendem Wasser, Alkohol, Aether, Chloroform, Kali-, Natronlauge und Ammoniak. Andererseits werden beide Substanzen langsam durch schwache Säuren wie Essigsäure, schweflige Säure, durch Chlorwasser, Alaunlösung, rasch durch stärkere Säuren wie Salz-, Sehwefel-, Salpetersäure entfärbt und zerstört. Beim Ultramarin entwickelt sich während der Säureeinwirkung Schwefelwasserstoff, der deutlich am Geruche erkennbar ist. Das konnte ich freilich mikrochemisch an den blauen Körnern nicht wahrnehmen. Weder war es mir möglich mikroskopische Gasbläschen mit Sicherheit auf die verschwindenden Körnchen zu beziehen, noch konnte ich Spuren von Schwefelwasserstoff durch deutliche Reactionen kenntlich machen. Ich verfuhy in der Weise, dass ich von Fliesspapier, welches mit einer Lösung von Bleiessig und einigen Tropfen Natronlauge getränkt war, einen Rahmen trocken auf den Objectträger legte. Auf das Fenster dieses Rahmens kam freischwebend ein Deckgläschen, das an seiner unteren Seite das Object in einem Tropfen Eisessig 
schwimmend trug, so dass der Tropfen weder den Objectträger berührte, noch sich auf dem Deckgläschen ausbreiten und so das Fliesspapier benetzen konnte. Ich hoffte so das in den kleinen Luftraum zwischen Objectträger, Deckgläschen und Fliesspapier eventuell sich ausbreitende $\mathrm{H}_{2} \mathrm{~S}$ an der Bräunung des letzteren kenntlich zu machen. Zugleich konnte ich die Zerstörung der blauen Körner mit Hartnack VIII gut beobachten. Ich kann dem stets negativen Resultat, das ich erhielt, keine grosse Bedeutung zuschreiben, da es unmöglich war, die der Reaction schädlichen Dämpfe der freien Säure vom Fliesspapier fern zu balten -- eine Benetzung desselben mit der Säure verhinderte die Reaction vollständig - und da ausserdem die betreffenden Mengen $\mathrm{H}_{2} \mathrm{~S}$ wohl sicher zu unbedeutend waren, um die Reaction zu erzeugen; Controlversuche mit Ultramarin lebrten mich wenigstens, dass erst bei einem Körnchen Ultramarin von Stecknadelkopfgrösse diese Reaction in gewünschter Deptlichkeit hervortrat. Um so wichtiger war es mir, noch eine andere, feinere Reaction auf Ultramarin anwenden zu können, die ich der Abhandlung von W. Stein, die Prüfung der Zeugfarben und Farbmaterialien (Eutin 1874, S. 52), verdanke. Dieser Autor giebt an, dass Ultramarin mit schwefelsaurem Kupferoxyd gekocht, schwarz wird; ich konnte dies an meinen verschiedenen Ultramarinproben bestätigen. Demgemäss fand ich nun auch, dass die blauen Körner, mit einem Tropfen einer dunkelblauen Lösung von Kupfervitriol gekocht, eine grauschwärzliche Farbe (wohl von Schwefelkupfer herrübrend) annahmen, während die Hornzellen grüngelblich gefärbt wurden. Diese Farben konnten durch kaltes und kochendes Wasser nicht ausgezogen werden; ein Tropfen Kalilauge, unter dem Deckglase zugesetzt, wandelte die grünliche Farbe der Hornzellen in violett, während die scbwarz gewordenen Körner schwarz blieben.

In Folgendem stelle ich die dem Ultramarin und den blauen Körnern der Comedoneń gemeinsamen Reactionen der Uebersicht wegen noch einmal tabellarisch zusammen, verhehle mir übrigens trotz der auffallenden Congruenz beider Reihen keineswegs, wie misslich es ist, beide einfach als gleichwerthig hinzunehmen. Denn die eine besteht aus chemischen Reactionen von zweifelloser Sicherheit, die andere ans mikrochemischen, deren Objecte oft an der Grenze der Wahrnehmung stehen und wegen der unmöglichen Iso- 
lirung aus ihrer organischen Umgebung zu Einwürfen und berechtigten Zweifeln Anlass geben.

Uitramarin

verändert sich auch beim
Kochen nicht $\left\{\begin{array}{c}\text { verändern sich auch beim } \\ \text { Kochen nicht }\end{array}\right.$
Die blaues Körner der Comedonen

auf Zusatz von:

destillirtem Wasser

Kalilauge $(1: 3)$

Natronlauge $(1: 3)$

Liq. ammon. caust.

Chloroform

Aether

absolutem Alkohol

Kochsalzlösung (1:10)

nimmt beim Kochen eine ( nehmen beim Kochen eine schwarze Farbe an $\left\{\begin{array}{l}\text { schwärzliche Farbe an } \\ \text { and }\end{array}\right.$ wird zersetzt, rascher beim f werden zersetzt, rascher Kochen, unter Abscheidung von $\mathrm{H}_{2} \mathrm{~S}$ u. $\mathrm{S}$ bein beim Kochen (unter $\mathrm{Ab}$ scheidung von $\mathrm{H}_{2} \mathrm{~S}$ u. S?) wird langsam in der Kälte

zersetzt
wird sofort zersetzt unter
Abscheidung von $\mathrm{H}_{2} \mathrm{~S}$ u, S $\left\{\begin{array}{c}\text { werden sofort zersetzt } \\ \text { (unter Abscheidung von } \\ \mathrm{H}_{2} \mathrm{~S} \text { u. S?) }\end{array}\right.$ werden langsam in der

zersetzt
wird sofort zersetzt unter
Abscheidung von $\mathrm{H}_{2} \mathrm{~S}$ u, S $\left\{\begin{array}{c}\text { werden sofort zersetzt } \\ \text { (unter Abscheidung von } \\ \mathrm{H}_{2} \mathrm{~S} \text { u. S?) }\end{array}\right.$ Kupfersulphat $(1: 10)$ Alaunlősung $(1: 10)$

Essigsäure $(1: 4)$

Aq. sulfurosa $\{$ Aq. chlori

Neben einer solchen Uebereinstimmung der chemischen Reactionen erscheint die des äusseren Ansehens der Körner mit dem käuflichen Ultramarin von geringerem Belange. Die Farbe der blauen Körner ist jedoch geradezu eine ultramarinblaue, gewöhnlich tief gesättigte; seltener kommt ein helles Himmelblau vor; niemals besitzt dasselbe einen Stich in's Gelbliche oder Grünliche. Die Körner sind weiter amorph wie das Ultramarin. Die ersten Ultramarinsorten, die ich mikroskopisch untersuchte, besassen sogar in der Grösse und Gestalt der Körner eine auffallende Uebereinstimmung mit den blauen Körnern der Comedonen. Ich fand jedoch bald, dass dieselben durch Stearin verfälscht waren, welches die feinen Körner zu gröberen zusammenbackte und dass die besten Ultramarinsorten ein viel feineres Pulver darstellen, welches durch Zerreiben in immer feineren Staub ohne bestimmte Form zerfällt. Es liegt daher der Gedanke nahe, dass das freie Fett, welches die Körner in den Comedonen nmgiebt, anf ihre Gestalt und ihr Aussehen auch nicht obne Einfluss ist. 
Das Voranstehende rechtfertigt, glaube ich, bereits die Annahme, dass es sich bei den blauen Körperchen entweder in der That um Ultramarin selbst oder wenigstens einen dem Ultramarin sehr ähnlichen Körper handelt, der in den meisten wesentlichen Eigenschaften mit ihm ubereinstimmt.

Da die Zusammensetzung des Ultramarins eine sehr complicirte und immer noch nicht mit Sicherheit ergründete und es noch keineswegs ausgemacht ist, dass die den verschiedenen Fabricationsweisen entspringenden Producte genau als identisch zu betrachten sind, würde es auch nicht wunderbar erscheinen, wenn einer oder der andere Bestandtheil durch einen chemisch nahverwandten Körper ersetzt werden könnte. Ich erwähne dies besonders, da die dem Ultramarin eigenthümliche Thonerde bisher nicht als Bestandtheil des menschlichen Organismus nachgewiesen worden ist, während die übrigen Bestandtheile des Ultramarins, namentlich die Kieselsäure und der Schwefel grade in den Hornsubstanzen bekanntlich sehr rejehlich vorkommen. Allzu grosses Gewicht ist jedoch auf diesen bisher noch fehlenden Nachweis bei der verschwindend kleinen Menge Tbonerde, die hier in Betracht kommt, wobl nicht zu legen.

Viel merkwürdiger ist es, dass ein künstliches Glühproduct hier bei Körpertemperatur synthetiseh entstehen soll und ich überlasse die etwaigen Schlussfolgerungen den Chemikern, ebenso wie ich die schwierigeren Fragen, woraus bestehen die schwarzen und braunen, in Königswasser löslichen Körperchen den Männern von Fach anheim geben muss.

Dass die letzteren sich weniger leicht in Säuren lösen wie die blauen Körner habe ich schon oben bemerkt. Im Uebrigen sind ihre Reactionen denen der blauen sehr ähnlich, indem sie von starken Säuren, besonders beim Kochen, rasch gelöst, dagegen von verdünnten und concentrirten Alkalien nicht angegriffen werden. Hierin zeigen sie auch dasselbe Verhalten wie die diffuse Färbung der Hornzellen des Comedonenmantels. Kocht man freilich ganze Comedonen längere Zeit in Kalilauge, so scheint es, als ob ebenfalls der schwarze Punkt an Intensität verlöre. Ich glaube jedoch, dass es sich dabei nur um ein physikalisches Phänomen handelt, indem nehmlich der Farbstoff beim Aufquellen der Comedonenspitze und aller einzelnen Zellen über eine grössere Oberfläche 
vertheilt wird. Durch längere Einwirkung von Chlorwasser in der Kälte wird die diffuse, schwarze Färbung erheblich lichter ohne ganz zu verschwinden, ähnlich wie dunkle Haare durch chlor gebleicht werden. Die durch dieses Mittel zum Theil entfärbten Comedonen zeigen in ihrem Innern sehr wenig schwarze Körner mehr, während braune und gelbliche noch ziemlich reichlich vorhanden sind (die blauen sind vollständig geschwunden). Es ist mir daher wahrscheinlich, dass bei der Einwirkung des Chlorwassers schwarze Körner in braune umgewandelt werden. Diese Analogie mit dem diffusen Haarpigment festhaltend, versuchte ich auch die in jenem Falle erprobte Aufhellung durch Wasserstoffsuperoxyd, welches den Hauptbestandtheil des sog. "golden hair wash" der Friseure ausmacht und zum Blondmachen dunkler Haare gebraucht wird. Es trat nach 24 stündiger Einwirkung dieses Wasserstoffsuperoxydpräparats denn auch eine der Chlorbleichung ähnliche Aufhellung des diffusen Comedonenpigments ein.

Dieses sind die wenigen chemischen Thatsachen, die ich bisher über das Verhalten der dunklen Körner und des diffusen Pigments gesammelt habe. Sie sprechen, denke ich, wenigstens dafür, dass diese Pigmente in ihrer Zusammensetzung den blauen Körnern einerseits, dem diffusen Haarpigment andererseits nicht so ferne stehen, die deshalb gegenseitig zur Aufklärung der chemischen Constitution herangezogen $\mathrm{zu}$ werden verdienen.

Es werden die betreffenden Untersuchungen wohl geeignet sein, die Chemie der Hornstoffe um einen Schritt zu fördern. Mir wenigstens will es scheinen, als ob in der uns hier beschäftigenden, mit Unrecht lange Zeit als Schmutz registrirten, diffusen Gelb-, Braun- und Schwarzfärbung nichts Anderes zu finden sei als in der Bräunung und Schwärzung, die wir in der diffusen Färbung der Haarzellen, an den Hörnern der Thiere und Menscben, an den Hufen und Klauen, an den Nägeln bei gewissen chronischen Krankheiten, in der Hornschicht bei Keratosis simplex und hochgradig bei Keratosis nigra antreffen. Ich hoffe, dass der Fund der blauen Körperchen, die eine verhältnissmssig leichte Deutung zulassen, ein Schritt und Wegweiser auf diesem Pfade sei und bescheide mich im Uebrigen, diese nicht nur für das Verständniss der Acne, sondern für die physiologische Chemie im Allgemeinen wichtigen Fragen hiermit angeregt zu baben. 
Ich kann diese Erörterungen nicht schliessen, ohne einige Fragen $z u$ berühren, die sich unmittelbar an die neue Auffassung des Comedonenpigments anreihen.

Erstlich wirft sich die Frage auf, ob die von den älteren Dermatologen mehr als von den neueren berücksichtigte, blaue Farbe mancher Comedonen in einem Abbängigkeitsverbältniss zu den von mir gefundenen blauen Körnern steht. Diese Frage kann ich mit Entschiedenheit verneinen. Für gewöhnlich sind die blauen Körner gewiss viel zu klein und spärlich gegenüber den braunen und schwarzen, um auf die Gesammtfarbe der Zone, in der sie liegen, einen Einfluss üben zu können. Aber auch wenn sie noch so reichlich vorhanden sind, werden sie ja vollständig verdeckt von der diffusen braunen, resp. schwarzen Färbung der Comedonenspitze. Nur diese letztere bestimmt für gewöhnlich die Farbe der Comedonen. Dagegen hatte ich in letzter Zeit genügende Gelegenheit, die Ursache der blauen Farbe gewisser Comedonen zu erforschen, besonders an einem älteren Herrn, der an erschlaffter Darmperistaltik litt und mit consecutiven Stauungserscheinungen an Füssen und Händen und Acne rosacea behaftet war. Die stark geröthete Nase desselben war im mittleren Theile mit blaugrauen Punkten besetzt, die der blauen Tättowirung durch Pulver täuschend ähnlich sahen, wie Alibert es seiner Zeit schon gut beschrieben bat.

Die Comedonen waren klein im Verhältniss zu den weiten Talgdrüsenmündungen, in denen sie sassen und von so viel flüssigem Fett umgeben, dass sie auf leisen, seitlichen Druck gleichsam herausglitschten und auf Papier gelegt einen grossen Fettfleck erzeugten. $\mathrm{Zu}$ meinem Erstaunen schwand die bläuliche Farbe schon fast ganz, als die Comedonen auf einem weissen Hintergrunde lagen und noch mehr, wenn man sie gegen das Licht hielt, wobei die Farbe nicht viel von der gewöbnlichen, gelblich abwich. Die mikroskopische Untersuchung zeigte überdies nur verhältnissmässig wenig blaue Körner in denselben. Dieser Farbenwechsel und damit die blaue Farbe einiger Comedonen scheint mir somit einfach auf das bekannte Phanomen binauszulaufen, dass trübe Medien im durchgelassenen Lichte röthlichgelb, im reflectirten bläulich erscheinen, welches von Goethe entdeckt, in einseitiger Weise als „Urphänomen " zur Begründung seiner Farbenlehre verwandt wurde ${ }^{1}$ ),

1) Zur Farbenlebre. X. 150-151. 
um später von Brücke seine richtige Deutung zu erhaiten. Nach Br ücke sind es kleine, differente Theilchen innerhalb eines Mediums, welche dieses trüb erscheinen lassen und durch Interferenz der an ihrer vorderen und hinteren Fläche gebrochenen Strahlen das Licht beim Durchgange und bei der Reflexion vollkommen auslöschen würden, wenn diese Theilchen unendlich klein wären. Weno dieselben dagegen nur sehr klein sind, so wird nur Licht von bestimmter Wellenlänge durch Interferenz aufgehoben und zwar bleiben dem durchgehenden Lichte Strahlen des rothen, dem reflectirten Lichte Strahlen des violetten Spectrumendes. Diese Erklärung passt für die mit viel flüssigem Fett getränkten Comedonen vortrefflich, da bier die durchsichtige Hornmasse sich mikroskopisch von allerfeinsten Fettkügelchen durchsetzt erweist, deren Brechungsvermögen von dem der Hornzellen bedeutend abweicht. Sie stellen demgemäss trübe Medien dar, welche aúf dem dunkeln Hintergrund der Follikel bläulich, im durchfallenden Lichte gelblich aussehen müssen.

Eine andere Frage, zum Theil bereits im Vorgehenden beantwortet, ist die therapeutische. Ich habe gezeigt, dass die schwarze, diffuse Färbung der Comedonen nur in Säuren löslich und zerstörbar ist. Hiermit stimmt die alltägliche Erfahrung, dass unter den gewöhnlichen Behandlungsmethoden der Acne wohl eine Abschwellung der Haut, eine Verengerung der erweiterten Hautgefässe zugleich mit einer allmählichen Verdünnung der Oberhaut und Eröffnung der Follikel, letzteres besonders bei der mechanischen Behandlung, erreicht wird; was jedoch die Comedonen anlangt, so sind wir fast allein auf die mechanische Behandlung, das Ausquetschen derselben, angewiesen. Die gewöhnlich gebrauchten Schwefelmittel vermehren sogar im Anfange der Behandlung die Anzabl der schwarzen Comedonenköpfe erheblich. Dasselbe sieht man häufig bei alleiniger Anwendung von Quecksilbermitteln, z. B. des grauen Quecksilberpflasters, bei vollständiger Fernbaltung - auch vorher und nachber - jeden Schwefelmittels. Ich beobachte es aber auch sogar seit längerer Zeit unter dem Gebrauche von Mitteln wie kieselsaure Thonerde, die man für völlig indifferent zu balten geneigt sein wird und auf deren Verwendung ich kam, um eben einen Ersatz für den Schwefel zu finden, wo ich zugleich die Anwendung von Blei - und Quecksilberpräparaten beabsichtigte. Das diesen Mitteln Gemeinsame ist eine für die Beseitignng der Acne in einer 
Beziehung sehr günstige Austrocknung der Oberbaut und des Papillarkörpers. Dass hierbei aber das Auftreten von neuen Comedonen begünstigt wird und die vorhandenen rasch an Alter, Festigkeit, Prominenz und Schwärze zunehmen, ist mit meiner Theorie, nach welcher die Entstehung aller Comedonen stets nur auf dem Verschlusse der Follikel durch abnorm feste Hornschicht berubt, auf's Beste vereinbar. Bis jetzt besitzen wir also eigentlich gegen Comedonen nur eine mechanische Behandlung; denn die Besserung die bei den genannten Mitteln allmählich auch eintritt, ist auf die allgemeine Abstossung der Oberhaut zurückzuführen und wenn diese auch durch chemische Wirkungen zu Stande kommt, kann man die indirecte Behandlung der Comedonen dabei doch nur als eine mechanische bezeichnen.

Dem gegenüber verfolge ich schon seit längerer Zeit, seit ich einmal früher durch Verwendung der essigsauren Thonerde auf den Nutzen der Säuren gegen Comedonen aufmerksam geworden, und bewusst, seit ich die Thatsache der Löslichkeit des Pigments in Säuren kennen lernte, die directe und chemische Inangriffnahme der Comedonen durcb Säuren, die mich bisher mehr befriedigte, als irgend eine andere Behandlung und sich vortrefflich mit jeder sonstigen Acnetherapie vereinigen lässt.

Ich verordne jetzt gewöhnlich:

$\begin{array}{ll}\text { Kaolini (Bol. alb.) } & \text { pp. IV } \\ \text { Glycerini } & \text { pp. III } \\ \text { Aceti } & \text { pp. II }\end{array}$

mit oder ohne Zusatz von etwas ätherischem 0el. Die Salbe wird Abends, wenn möglich auch Tags über, eingerieben. Die Augen miissen während des Einreibens geschlossen werden. Die stereotype Angabe der Patienten ist, dass schon nach wenigen Tagen die Comedonen sämmtlich leichter auszudrücken seien, dass die meisten derselben durch Waschen mit Bimsteinseife bereits abgerieben werden. Objectiv ist wahrzunehmen, dass das Neuauftreten schwarzer Punkte mit einem Male aufhört und dass die vorhandenen in kurzer Zeit abblassen und verchwinden, ohne dass man sich die Mühe giebt, sie auszudrücken. Viele chronische Acnepatienten, die in früheren Jahren für das Emplastrum bydrargyri, für Schwefelkalkpomade, für Waschwässer mit Campher und Schwefel oder Quecksilberalbuminat 
eingenommen waren, wissen die Vorzüge dieser einfachen und billigen Salbe nicht hoch genug anzuschlagen.

Es versteht sich von selbst, dass man denselben Effect in Bezug auf die schwarzen Köpfe der Comedonen erreicht durch länger fortgesetztes Einpinseln von Essig, Aufstreichen von Citronensaft; ja der Arzt selbst kann ihn durch Bepinseln mit verdüunter Salzsäure in kürzester Zeit zu Stande bringen. Sehr gut vereinbar sind mit dieser Salbe die Schwefelmittel einerseits, die Blei- oder Quecksilberhaltigen andererseits. Ausgeschlossen sind dagegen die alkalischen Mittel, auch die so populär gewordene Zeissl'sche Schwefelpaste mit Kali carbonicum, die in Uebrigen recht gute Dienste leistet, in Bezug auf die Comedonen jedoch theoretisch und practisch in diametralem Gegensatze zu unserer Therapie sich befindet. Einfache Seifenwaschungen mit ihrer vorübergehenden Wirkung sind dagegen von derselben nicht ausgeschlossen, während Umschläge von Seife bekanntlich den alkalischen Mitteln gleichwerthig sind.

Es ist aber nach meiner Ansicht ein Vorurtheil, dass eine beschleunigte Abstossung der Oberhaut stets am einfachsten und besten durch Alkalien, resp. Seifen zu bewerkstelligen sei. Verdünnte Lösungen von Salzen und Säuren, nur mit derselben Consequenz angewandt, bewirken die Abschiebung der Hornschicht ebenso prompt, freilich in anderer Weise. Während die Alkalien die Hornschicht zur Mortification bringen unter Aufquellung der Zellindividuen und dadurch, bis die Abstossung (Pityriasis lamellosa) vollendet, der Zusammenhalt der Hornzellen mit allen seinen Consequenzen (u. A. Bildung neuer Comedonen) eine Zeit lang künstlich erhöht ist, werden die Horuzellen durch Säuren und Salze obne Aufquellung zum Absterben gebracbt. Daher hier der Zusammenhang der Hornzellen noch rascher als gewöhnlich aber sonst in normaler Weise nachgiebt (Pityriasis furfuracea vel Desquamatio insensibilis) und somit von vornberein die beabsichtigte Wirkung, die Freilegung der Drüsenmündungen, zu Tage tritt. Im Uebrigen haben die verdünnten Säuren auch keinen schädlichen, sondern eher günstigen Einfluss auf die Blutüberfüllung des oberflächlichen Gefässnetzes der Cutis, besonders der Gefässkränze um die Talgdrüsen, ein Punkt, der überhaupt nicht schwer in's Gewicht fällt, da mit der Eröffnung der Follikel die Ursache der Stauungs- und Entzündungserscheinungen wegfällt. 
Die Wirkung der Säure wäre also auf die zwei Hauptmomente zurückzuführen: sie wirkt erstens direct kosmetisch, indem sie die schwarze Färbung in braun und gelb verwandelt und allmählich zerstört und hierin wird sie von keinem andersartigen Mittel erreicht und zweitens erzeugt sie eine stärkere Abschuppung der Hornschicht, welche die Comedonen lockert und die Drüsenmündungen freilegt.

Ich bin durchaus nicht der Meinung, die Säuren äusserlich (innerlich sind sie in früherer Zeit viel gegeben worden) zuerst angewandt zu haben, aber ich finde nirgendwo eine theoretische oder practische Bemerkung darüber in den gangbaren Lehrbüchern. Die neuesten (Kaposi, Behrend) geben nur die gewöhnlichen Schwefelmittel mit Alkalien oder Seifen oder die Anweisung Schwefelmittel und Alkalien abwechselnd zu gebrauchen. Ich glaube, nach dem Gesagten, die Anwendung der Säuren, insbesondere in Verbindung mit austrocknenden Mitteln (Schwefel, Bolus) und selbstverständlich der chirurgischen Behandlung (Schaben, Sticheln) angelegentlichst empfehlen zu dürfen sowohl gegen Comedonen wie gegen Acne überbaupt, die nach meiner Meinung in allen neueren Lehrbüchern zu sebr getrennt von den Comedonen abgehandelt wird, da sich die Acne zu den Comedonen verhält, wie die Krankheit zu einem nothwendigen, secundären Krankheitsproduct, das ausser bei Acne nur noch auf Narben eine bescheidene und zufällige Existenz fristet. 